УДК 621.45 .038

\title{
ПЕРСПЕКТИВНЫЕ СХЕМЫ ПЛЕНОЧНОГО ОХЛАЖДЕНИЯ: ВЛИЯНИЕ ВНЕШНЕЙ ТУРБУЛЕНТНОСТИ
}

\author{
Халатов А.А.', академик НАН Украины, Борисов И.И., канд. техн. наук, \\ Дашевский Ю.Я.․, канд. техн. наук \\ ${ }^{1}$ Институт технической теплофизики НАН Украины, ул. Желябова, 2а, Киев, 03680, Украина \\ ${ }^{2}$ НПКГ «Зоря» - «Машпроект», просп. Жовтневый, 42а, г. Николаев, 54018, Украина
}

Наведено результати експериментальногодослідженнявпливузовнішньої турбулентності (7 \%) на ефективність плівкового охолодження пласкої поверхні за допомогою похилих отворів у заглибинах різної форми - кратерах, траншеї, напівсферичних сегментах. Показано, що на відміну від отворів без заглибин, вплив даного фактору є незначним.
Представлены результаты экспериментального исследования влияния внешней турбулентности (7 \%) на эффективность пленочного охлаждения плоской поверхности с помощью наклонных отверстий, расположенных в углублениях различной формы - кратеpax, траншее, полусферических сегментах. Показано, что в отличие от отверстий без углублений, влияние данного фактора незначительно.
Results of experimental study of the effect of external turbulence $(7 \%)$ on the film cooling efficiency over the flat plate with coolant supply into shallow indentations of different shape namely craters, trench, semispherical segments, were presented. It is shown that the effect of external turbulence for the investigated schemes is relatively low in comparison with holes without indentations.

Библ. 13, рис. 3.

Ключевые слова: пленочное охлаждение, отверстия в углублениях, адиабатная эффективность, внешняя турбулентность.

$d$ - диаметр отверстия;

$h$ - глубина кратера, траншеи, полусферического сегмента;

$m$ - параметр вдува;

$t-$ поперечный шаг расположения отверстий;

$x$ - продольная координата;

\section{Введение}

Способы пленочного охлаждения, обеспечивающие высокую эффективность и равномерность покрытия поверхности, представляют большой научный и практический интерес для исследователей и разработчиков систем охлаждения лопаток и камер сгорания высокотемпературных газовых турбин. Наряду с использованием фасонных отверстий [1], позволивших поднять на качественно новый уровень характеристики перфорационного пленочного охлаждения и приблизивших их к показателям для сплошной щели, в последние годы предложен ряд перспективных и более технологически простых решений, основанных на подаче охладителя в мелкие (с отношением глубины к диаметру порядка $0,5 \ldots 1,0)$ углубления различной формы - кратеры, траншею, полусферические сегменты [2-4]. Данный подход базируется на том, что охладитель перед выходом на поверхность успевает распространиться в поперечном направлении, что существенно снижает отрывные явления и интенсивность вторичных парных вихрей, ухудшающих защитные свойства пленочного охлаждения.

Для реальных условий течения в проточном тракте турбины характерно наличие ряда факторов, влияющих $z$ - поперечная координата;

$\mathrm{Tu}$ - степень турбулентности;

$\alpha$ - угол наклона отверстия;

$\bar{\eta}$ - средняя по ширине эффективность пленочного охлаждения.

на характеристики пленочного охлаждения. К числу основных относится внешняя турбулентность, типичный уровень которой при обтекании лопаток (исключая входную кромку) составляет, по данным [5], 4...12 \%. Внешняя турбулентность в различных системах вдува проявляется по-разному.

Для щелевого вдува повышение степени турбулентности приводит к существенному снижению эффективности, начиная от начального участка, причем, с увеличением продольной координаты и уменьшением параметра вдува влияние турбулентности возрастает. По данным [6], для наклонной щели $\left(\alpha=20^{\circ}\right)$, в конце рабочего участка $(x / s \approx 100)$ при минимальном параметре вдува $(m=0,5)$ увеличение значения Тu с $3 \%$ до $12 \%$ и $22 \%$ снижает эффективность на $30 \%$ и $50 \%$ соответственно. Значение параметра вдува в этих исследованиях изменялось от 0,5 до 1,8 . В экспериментах [7] с такой же наклонной щелью, выполненных в диапазоне значений параметра вдува $0,2 \ldots 1,4$, получено максимальное снижение эффективности на $35 \%$ при Тu $=8 \%$, и на $55 \%$ - при Тu $=14 \%$.

Исследования [8-10] по влиянию внешней турбулентности на эффективность пленочного охлаждения однорядной системой наклонных отверстий показали, 
что оно зависит от параметра вдува. При малых его значениях $(m<1)$ внешняя турбулентность снижает среднюю по ширине эффективность пленочного охлаждения, однако при $m>1,5$ имеет место некоторое ее повышение. Так, по данным [10], для цилиндрических отверстий при возрастании степени турбулентности от 3,6 \% до $11 \%$, при малых и умеренных параметрах вдува средняя по ширине адиабатная эффективность снижалась до 25 \%, а при высоких - наблюдался незначительный ее прирост, вследствие улучшения перераспределения охладителя в поперечном направлении и уменьшения влияния отрыва.

Для рядов фасонных отверстий влияние турбулентности качественно ближе к случаю сплошной щели, чем к цилиндрическим отверстиям. С увеличением степени турбулентности основного потока эффективность снижается при всех значениях параметра вдува, однако с его увеличением влияние данного фактора ослабевает.

Различный характер влияния турбулентности на эффективность для цилиндрических и фасонных отверстий проанализирован в [5]. Для случая цилиндрических отверстий с повышением параметра вдува повышение степени турбулентности приводит к уменьшению протяженности зоны отрыва потока вследствие интенсификации перемешивания охладителя. Кроме того, независимо от параметра вдува, высокая турбулентность улучшает поперечное распределение охладителя, оба эти фактора приводят к повышению эффективности. Для истечения струи охладителя из фасонных отверстий веерного типа отсутствует или слабо выражена зона отрыва, поэтому турбулентность лишь интенсифицирует смешение охладителя и основного потока, снижая эффективность. Кроме этого, фасонные отверстия оказались более чувствительными к уровню турбулентности, чем цилиндрические, поскольку из-за большей ширины струи они имеют большую площадь взаимодействия охладителя с основным потоком.

По данным [5], для веерных отверстий при увеличении степени турбулентности основного потока от $2 \%$ до 7,5 \% средняя по ширине эффективность пленочного охлаждения снижается на $30 \%$ при $m=0,5$, и до $15 \%-$ при $m=2,5$. В [11] получено, что для значения параметра вдува $m=1,5$ при повышении степени турбулентности с 0,5 \% до 13,2 \% средняя по поверхности $(2<x / d<22)$ эффективность пленочного охлаждения системой фасонных отверстий снижается на $17 \%$. Эти данные согласуются с данными работы [10], в которой для такого же пространственного интервала увеличение уровня турбулентности приводило к снижению эффективности на $11 \%$.

Для систем наклонных отверстий в углублениях влияние внешней турбулентности практически не изучено. Имеются результаты численных исследований для однорядной системы фасонных отверстий в траншее [12] до значений параметра вдува $m=1,3$. Края отверстия были «вписаны» в траншею. По сравнению с вариантом фасонных отверстий без траншеи, чувствительность эффективности охлаждения к степени турбулентности основного (внешнего) потока заметно снижается. При значениях параметра вдува $m>1,0$ данный фактор повышает эффективность охлаждения на начальном участке.

Целью настоящей работы является сравнительное экспериментальное исследование влияния внешней турбулентности на эффективность пленочного охлаждения при подаче охладителя в кратеры, траншею и полусферические углубления в широком диапазоне изменения параметра вдува - от 0,5 до 2,0.

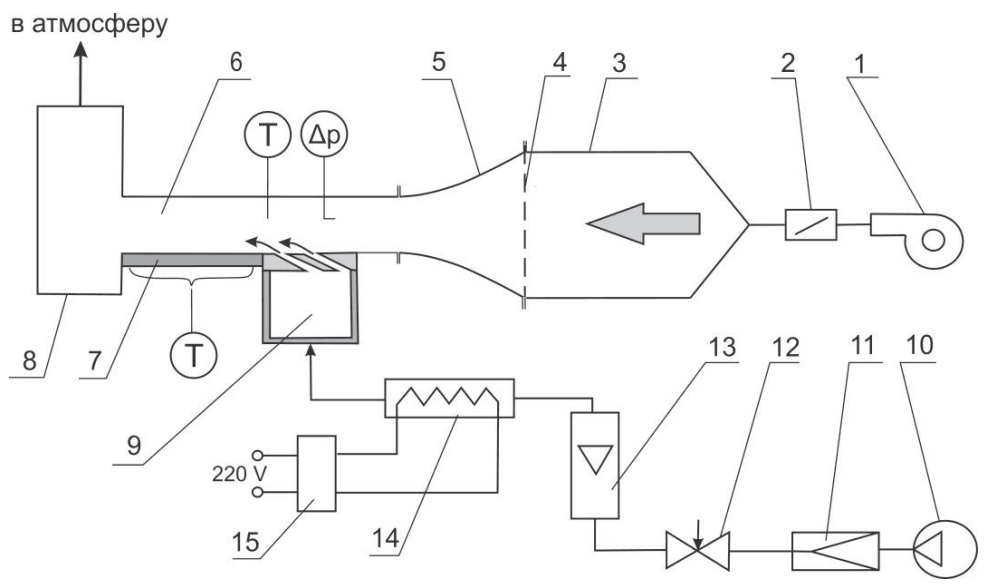

Рис. 1. Схема экспериментальной установки.

1 - вентилятор; 2 - поворотный затвор; 3 -ресивер; 4 -турбулизирующая решетка;

5 - сопло Витошинского; 6 - рабочий участок; 7 -адиабатная пластина; 8-успокоитель; 9 -участок вдува вторичного воздуха; 10 -компрессор; 11 - фильтр-регулятор; 12 - вентиль; 13 - ротаметр; 14 - омический нагреватель вторичного воздуха; 15 - источник питания постоянного тока. 


\section{Экспериментальная установка и методика проведения измерений}

Эксперименты проводились на аэродинамической трубе открытого типа (рис. 1), работающей от центробежного вентилятора ВВД-5. Внешний (основной) поток воздуха от вентилятора подавался в ресивер и далее поступал в рабочий участок. Равномерное по сечению поле скорости на входе в рабочий участок обеспечивалось установкой на выходе из ресивера сопла, спроектированного по профилю Витошинского. После рабочего участка воздушный поток выходил в успокоительную емкость и далее - в атмосферу. Для исследования эффективности пленочного охлаждения выбрано обратное направление теплового потока: основной поток - холодный, а вторичный - нагретый. Вдуваемый (вторичный) воздух от индивидуального компрессора через регулирующий вентиль, расходомер (ротаметр) и электронагреватель подавался в рабочий участок через отверстия вдува.Рабочий участок представляет собой плоский канал с поперечным сечением 294х34 мм, соответствующим выходному сечению сопла Витошинского. Нижняя стенка рабочего участка включает пластину из оргстекла с отверстиями вдува и адиабатную пластину из асбоцемента $(\lambda=0,17 \mathrm{BT} / \mathrm{M} \cdot \mathrm{K})$. В пластину вмонтирован ряд из девяти хромель-алюмелевых термопар, расположенных на одной линии в продольном направлении с постоянным по длине шагом (10 мм). В установке предусмотрена возможность перемещения пластины в поперечном направлении.

Для генерации турбулентности использовалась решетка (перфорированная пластина) с относительным проходным сечением 17,3\%. Она устанавливалась на входе в сопло Витошинского, в этом случае на выходе из сопла обеспечивается изотропная турбулентность [13]. Уровень турбулентности потока в рабочем участке перед отверстиями вдува измерялся термоанемометром постоянной температуры, и в настоящих экспериментах составлял около 7 \%. Без решетки входная турбулентность потока составляла около $1 \%$.

В экспериментах измерялись скоростной напор основного потока, температура основного потока, температура и расход вдуваемого потока, температура адиабатной поверхности в различных точках по длине пластины.

Параметры экспериментов были следующими: скорость основного потока воздуха $35 \ldots 37 \mathrm{~m} / \mathrm{c}$, его температура $25 \ldots 33{ }^{\circ} \mathrm{C}$, температура вдуваемого воздуха - от 65 до $80^{\circ} \mathrm{C}$; отношение плотностей вдуваемого и основного потока составляло $0,84 \ldots 0,87$; параметр вдува $m$ изменялся от 0,5 до 2,0. Измерение профиля скорости основного потока в пристеночном слое перед отверстиями подачи вторичного воздуха показало, что пограничный слой близок к турбулентному (значение формпараметра составляло величину около 1,4$)$.

Исследованные системы вдува показаны на рис. 2.

Диаметр отверстий $d$ во всех исследованных вариантах составлял 3,2 мм, угол наклона $\alpha$ осей отверстий к поверхности составлял $30^{\circ}$. Поперечный шаг отверстий для однорядных вариантов (кратеров и траншеи) составлял $t=10$ мм $(t / d \approx 3,0)$. Диаметр кратеров $D$ и ширина траншеи $B$ соответствовали размеру эллипса наклонного отверстия, т.е. были «вписаны» в кратер или траншею. Глубина кратеров и траншеи была одинаковой $h=2,4$ мм $(h / d \approx 0,75)$. Относительная длина отверстий вдува $L / d$ составляла: 6,25 для отверстий в кратерах и траншее, и 4,4 - для отверстий в сферических углублениях. Продольное расстояние « $x »$ отсчитывалось от «среза» отверстия, поперечное расстояние «z» отсчитывалось от центральной линии одного из отверстий. В двухрядной системе отверстий в сферических углублениях диаметр углубления $D$ составлял 8 мм, глубина $h=4 \mathrm{мm}(h / D=0,5)$, поперечный шаг углублений (отверстий) $t=16$ мм $(t / d=5)$, а продольный $t_{1}=8$ мм $\left(t_{1} / d=2,5\right)$.

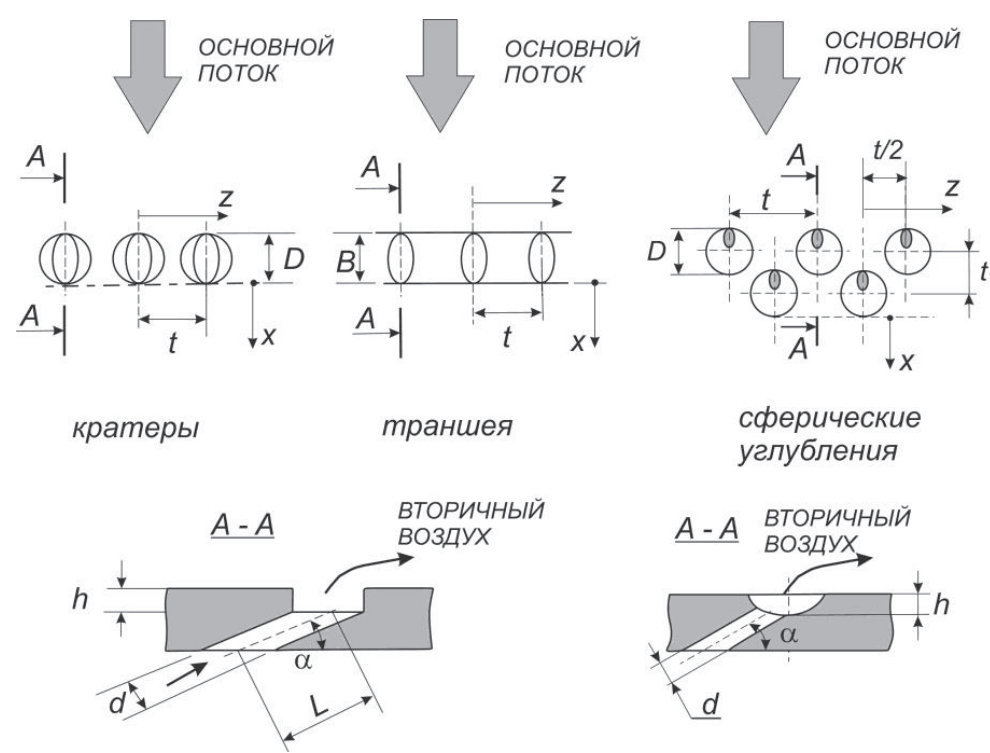

Рис. 2. Исследованные схемы пленочного охлаждения. 
Результаты и обсуждение

Результаты по влиянию турбулентности на эффективность пленочного охлаждения в виде зависимости фактора изменения эффективности от относительной продольной координаты для систем отверстий в углублениях показаны на рис. 3 .

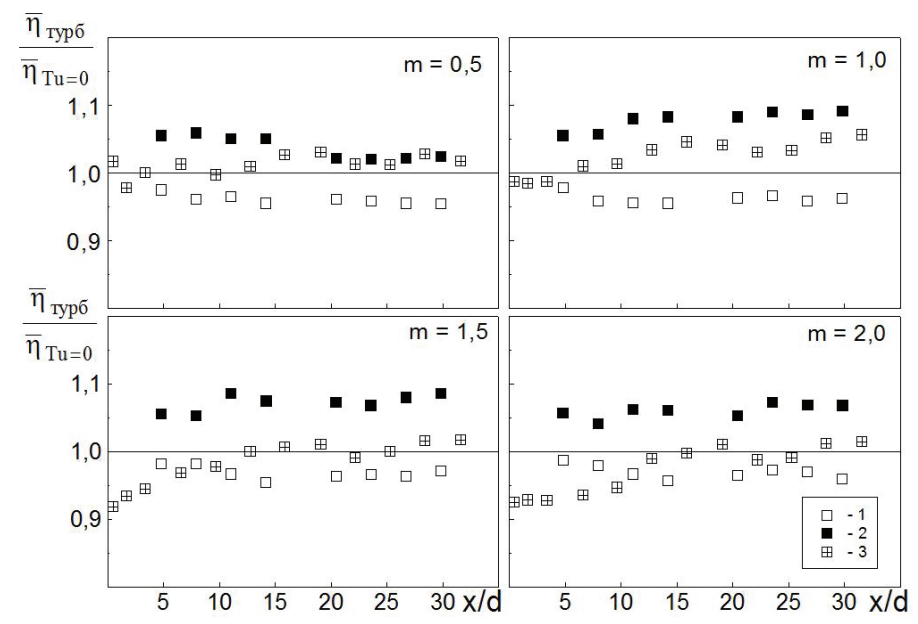

\section{Рис. 3. Влияние степени турбулентности основного потока $($ Ти $=7 \%)$ на осредненную в поперечном направлении эфрективность пленочного охлаждения. \\ 1 - однорядная система отверстий в кратерах; 2 - однорядная система отверстий в траншее; 3 - двухрядная система отверстий в полусферических углублениях.}

Как видно из рисунка, для исследованных в настоящей работе схем влияние турбулентности проявляется по-разному, однако в целом можно отметить, что в отличие от систем цилиндрических и фасонных отверстий, это влияние выражено значительно слабее. Данный факт можно объяснить дополнительной турбулизацией струй при ударе о кромку кратеров или траншеи, а также взаимодействием струй от соседних рядов для двухрядной системы отверстий в полусферических углублениях. Перечисленные эффекты снижают влияние внешней турбулентности.

Для однорядной конфигурации отверстий в кратерах турбулентность основного потока (до 7 \%) незначительно, на $3 \ldots 4 \%$, снижает среднюю по ширине поверхности эффективность пленочного охлаждения на основном участке вдува $(x / d>10)$, а на начальном участке ее влияние выражено слабее.

Для траншейной конфигурации внешняя турбулентность увеличивает эффективность охлаждения на $5 \ldots 8 \%$, в отличие от других схем пленочного охлаждения с безотрывным обтеканием поверхности пленкой охладителя - тангенциальной щели и фасонных отверстий - для которых турбулизация потока приводит к снижению эффективности. Этот результат согласуется с данными работы [12], авторы которой объясняют рост эффективности дополнительной дисперсией струй, которая способствует прижатию охладителя к поверхности. Внешняя турбулентность способствует лучшему распределению вторичного воздуха по траншее.Для двухрядной системы полусферических углублений при значении параметра вдува $m=0,5$ влияние внешней турбулентности проявляется слабо, и изменение эффективности охлаждения практически находится в пределах погрешности эксперимента. При $m=1,0$ внешняя турбулентность незначительно (на $3 . .4 \%$ ) повышает эффективность при $x / d>10$. При $m=1,5$ и $m=2.0$ внешняя турбулентность снижает эффективность пленочного охлаждения на начальном участке $(x / d<15)$ примерно на $8 \%$, а далее вниз по потоку ее влияние практически отсутствует.

Помимо результатов по средней эффективности, можно также отметить некоторые данные по локальной эффективности. Как показали эксперименты, наличие внешней турбулентности приводит к исчезновению неустойчивости на начальном участке вдува, которое наблюдалось в [4] для нетурбулизированного потока на линиях, проходящих по координате «x» через центры отверстий. По-видимому, это связано с тем, что турбулентность подавляет вторичные структуры, возникающие при обтекании сферических углублений. В противовес этому эффекту, на линии, проходящей по координате « $x\rangle$ между углублениями 1-го и 2-го ряда т.е. при значении поперечной координаты $z=0,25 t$, при турбулизации потока получено увеличение эффективности. Последний эффект можно объяснить улучшением поперечного распределения вторичного воздуха. В результате этих двух противоположных воздействий, на основном участке влияние турбулентности на осредненную в поперечном направлении эффективность охлаждения становится менее существенным.

\section{Выводы}

Выполненные экспериментальные исследования влияния внешней турбулентности (до $\mathrm{Tu}=7 \%$ ) на эффективность пленочного охлаждения с помощью систем наклонных отверстий в углублениях показали, что, 
в отличие от систем цилиндрических и фасонных отверстий, оно незначительно. Для однорядных систем отверстий в кратерах эффективность снижается на $3 \ldots 4$ \%, для траншейной схемы имеет место повышение эффективности (до 8 \%), а для двухрядной системы отверстий в полусферических углублениях турбулентность не оказывает влияния на основном участке, и снижает эффективность на начальном.

\section{ЛИТЕРАТУРА}

1. Bunker R.S. A review of shaped hole turbine filmcooling technology // Journal of Heat Transfer. -2005. V.127, No 4.- P.441-453.

2. Lu Y., Dhungel A., Ekkad S.V., Bunker R.S. Film cooling measurements for cratered cylindrical inclined holes // Journal of Turbomachinery. 2009. -Vol. 131, No 1. P.011005.

3. Lu Y., Dhungel A., Ekkad V., Bunker R.S. Effect of trench width and depth on film cooling from cylindrical holes embedded in trenches // Journal of Turbomachinery.2009. - Vol. 131, No.1. - P. 011003.

4. Халатов А.А., Борисов И.И., Коваленко А.С., Дашевский Ю.Я., Шевиов С.В. Эффективность пленочного охлаждения плоской поверхности системой наклонных отверстий, расположенных в сферических углублениях // Промышленная теплотехника. - 2012. - Т.34, №.3. C.5-12.

5. Saumveber C., Schulz A. Free-stream effects on the cooling performance of cylindrical and fan-shaped cooling holes // ASME Paper GT2008-51030.- pp. 879-891, doi: 10.1115/ GT2008-51030.

6. Carlson L.W., Talmor E. Gaseous film cooling at various degrees of hot gas acceleration and turbulence levels // International Journal of Heat and Mass Transfer. - 1968. V. 11, No 11. - P. 1695-1713.

7. Халатов А.А., Авраменко А.А., Борисов И.И. Газовая завеса на выпуклой поверхности с внешней турбулентностью и отрицательным градиентом давления // Теплофизика высоких температур. - 1991. - Т. 29, №1 . C.101-107.

8. Bons J.P., MacArthur C.D., Rivir R.B. The effect of high freestream turbulence on film cooling effectiveness // Journal of Turbomachinery. - 1996. - V.118, No4. - pp. 814-825.

9. Mayhew J.E. Baughn J.W., Byerley A.R. The effect of freestream turbulenceonfilm cooling adiabaticeffectiveness// International Journal of Heat and Fluid Flow. - 2003. - No 24. - pp. 669-679.

10. Saumveber C., Schulz A., Wittig S. Free-stream turbulence effects on film cooling with shaped holes // Journal of Turbomachinery. - 2003. - Vol.125, No 1.pp .65-73.

11. Schroeder R.P., Thole K.A. Effect of high freestream turbulence on flowfields of shaped film cooling holes // Journal of Turbomachinery. - 2016. - Vol. 138, No9. pp. 091001, 10p.

12. Baheri S., Jubran B.A., Alavi Tabrizi S.P. The effect of turbulence intensity on film cooling of gas turbine blade from trenched shaped holes // Proceedings of ASME Paper GT2008-50318. - pp. 301-310. doi:10.1115/GT2008-50318

13. Дыбан Е.П., Эпик Э.Я. Теплообмен и гидродинамика турбулизированных потоков. К.: Наукова думка, 1985. $-294 \mathrm{c}$. 


\section{ADVANCED FILM COOLING SCHEMES: EFFECT OF EXTERNAL TURBULENCE}

\author{
Khalatov A.A. ${ }^{1}$, Borisov I.I. ${ }^{1}$, Dashevskyy Y.Y. ${ }^{2}$
}

${ }^{1}$ Institute for Engineering Thermophysics NAS of Ukraine, 2a Zhelyabov str., Kyiv, 03680, Ukraine

${ }^{2}$ Gas Turbine Research \& Production Complex «Zorya»«Mashproekt»,

42a prosp. Zhovtnevyy, Mykolaiv City, 54018, Ukraine

The aim of this paper is the comparative experimental investigation of the effect of external turbulence on the film cooling efficiency over the flat plate with coolant supply into shallow indentations of different shape namely craters, trench, semispherical segments. The experiments were carried out in open wind tunnel, in the wide range of blowing parameter - from 0,5 to 2,0. The perforated grid installed in front of the Witoszynsky nozzle provided the isotropic turbulence rate of $7 \%$ at the test section inlet. The experiments show the effect of external turbulence on the efficiency of investigated schemes is relatively low in comparison with cylindrical and shaped holes. For one-row crater configuration the efficiency is decreased by $3 \ldots . \%$, for the trenched scheme the efficiency is increased (up to $8 \%$ ), for the two-row scheme of semi-spherical indentations the external turbulence reduces the efficiency in the area after the holes, but doesn't influences the efficiency at the main area of film cooling.

References 13, figures. 3 .

Key words: film cooling, holes in indentations, adiabatic efficiency, external turbulence

1. Bunker R.S. A review of shaped hole turbine filmcooling technology. Journal of Heat Transfer. 2005. V.127, No 4. P.441-453.

2. Lu Y., Dhungel A., Ekkad S.V., Bunker R.S. Film cooling measurements for cratered cylindrical inclined holes. Journal of Turbomachinery. 2009. Vol. 131, No 1. P.011005.

3. Lu Y., Dhungel A., Ekkad V., Bunker R.S. Effect of trench width and depth on film cooling from cylindrical holes embedded in trenches. Journal of Turbomachinery. 2009. Vol. 131, No.1. P. 011003.

4. KhalatovA.A., Borisov I.I., Kovalenko A.S. Dashevskyy Y.Y, Shevtsov S.V. Effektivnost plionochnogo ochlazhdenyja ploskoi poverchnosti systemoj naklonnych otverstij raspoloxhennych $\mathrm{v}$ sfericheskich uglublenijach [Film cooling efficiency over the flat plate by inclined holes in spherical indentations]. Promyshlennaja teplotechnika. 2012. V.34, №.3. P.5-12.

5. Saumveber C., Schulz A. Free-stream effects on the cooling performance of cylindrical and fan-shaped cooling holes. ASME Paper GT2008-51030. pp. 879-891, doi: 10.1115/ GT2008-51030.

6. Carlson L.W., Talmor E. Gaseous film cooling at various degrees of hot gas acceleration and turbulence levels. International Journal of Heat and Mass Transfer. 1968. V. 11, No 11. P. 1695-1713.

7. Khalatov A, Avramenko A, Borisov I. Gas screen for a convex surface with external turbulence and a negative pressure gradient. High temperature. 1991. Vol. 29, No 1. P.96-102.

8. Bons J.P., MacArthur C.D., Rivir R.B. The effect of high freestream turbulence on film cooling effectiveness. Journal of Turbomachinery. 1996. V.118, No4. pp. 814-825.

9. Mayhew J.E. Baughn J.W., Byerley A.R. The effect of freestream turbulence on film cooling adiabatic effectiveness. International Journal of Heat and Fluid Flow. 2003. No 24. pp. 669-679.

10. Saumveber C., Schulz A., Wittig S. Free-stream turbulence effects on film cooling with shaped holes. Journal of Turbomachinery. 2003. Vol.125, No 1. pp .65-73.

11. Schroeder R.P., Thole K.A. Effect of high freestream turbulence on flowfields of shaped film cooling holes. Journal of Turbomachinery. 2016. Vol. 138, No9. pp. 091001, 10p.

12. Baheri S., Jubran B.A., Alavi Tabrizi S.P. The effect of turbulence intensity on film cooling of gas turbine blade from trenched shaped holes. Proceedings of ASME Paper GT2008-50318. - pp. 301-310. doi:10.1115/GT200850318

13. Dyban E.P., Epik E.Y. Teploobmen i gidrodinamika turbulizirovannych potokov [Heat transfer and hydrodynamic of turbulized flows]. Kiev. Naukova dumka, 1985. 294 p.

Получено 22.03.2017 Received 22.03.2017 